\title{
Orthosurgical management of severe class III malocclusion - A case report
}

\author{
Brig Anil Dhingra', Maj Pankaj Juneja ${ }^{2}, *$ \\ ${ }^{1}$ Consultant, ${ }^{2}$ Classified Specialist, Dept. of Orthodontics and Dentofacial Orthopedics, Command Military Dental Centre \\ (Northern Command), Udhampur, Jammu \& Kashmir, India \\ *Corresponding Author: Maj Pankaj Juneja \\ Email: drpankajjuneja1983@gmail.com
}

\begin{abstract}
Class III malocclusions are the result of insufficient forward growth of the maxilla, excessive forward mandibular growth or a combination of both. For patients whose orthodontic problems are so severe that neither growth modification nor camouflage offers a solution, surgery to realign the jaws or reposition dentoalveolar segments is the only possible treatment. Dramatic progress in recent years has made it possible for combined surgical orthodontic treatment to be carried out successfully for patients with a severe dentofacial problem. This case report highlights the successful management of a 19-year-old male with skeletal class III relationship with compromised esthetics and poor functional adaptation with combined orthodontic and surgical line of treatment. Patient's malocclusion was decompensated first by pre-surgical orthodontics with extractions of four pre molars followed by orthognathic surgery to achieve structural balance, acceptable esthetics and stable functional occlusion.
\end{abstract}

Keywords: Skeletal Class III malocclusion, Orthognathic surgery, Decompensation.

\section{Introduction}

In contemporary surgical-orthodontic treatment, a fixed orthodontic appliance has three uses. First, to accomplish the tooth movement needed in preparation for surgery; second, to stabilize the teeth and basal bone at the time of surgery and third, to allow the necessary post surgical tooth movement. Advances in surgical techniques have improved the predictability of favourable outcomes for patients undergoing orthognathic surgery. The correction of any dentoskeletal malocclusion always has a threefold goal of achieving functional efficiency, structural balance, and aesthetic harmony. ${ }^{1,2}$

Class III malocclusions are generally considered one of the most complex and difficult orthodontic problems to diagnose and treat. Class III malocclusion, though less prevalent than other phenotypes, expresses in a more severe form. Prevalence of class III malocclusion in Caucasians ranges from 0.8 to $4.0 \%$ and upto $12 \%$ in Chinese and Japanese populations. ${ }^{3-5}$

Orthognathic surgery and Orthodontic therapy go hand in hand with each other in these types of cases to achieve the desired results. ${ }^{6}$ Proper clinical examinations followed by the right diagnosis and treatment planning are the key to success in such cases.

\section{Case Report}

A 19-year-old male patient reported with the chief complaint of forwardly placed lower front teeth \& jaw and inability to talk properly. On examination, he was diagnosed as having severe skeletal class III malocclusion, having prognathic mandible, retrognathic maxilla, concave profile, increased lower facial height and reverse overbite with increased reverse overjet (Fig. $1)$. There was no relevant medical and family history. Radiographic examination confirmed the clinical findings (Fig. 2).

\section{Problem List}

1. Prognathic mandible.

2. Retrognathic maxilla.

3. Increased lower facial height.

4. Class III incisor and molar relationship.

5. Crowding $\mathrm{U} / \mathrm{L}$ arches.

6. Proclination $\mathrm{U} / \mathrm{L}$ anteriors.

7. Concave profile.

8. Compromised smile esthetics.

\section{Treatment Goals}

1. To address the skeletal discrepancy.

2. To decrowd in both the upper and lower arches.

3. To achieve class I molar and canine relation bilaterally.

4. To correct proclination of U/L incisors.

5. To achieve normal overjet and overbite.

6. To improve his profile and smile esthetics.

\section{Treatment Plan}

Combined Orthodontic and Surgical line of treatment, involving three phases:

1. Pre-surgical Orthodontics phase: It involved fixed mechanotherapy using 0.022 MBT prescription pre-adjusted edgewise appliance with extraction of all four first bicuspids, over retained 85 and root stump 65. Extraction space was utilized for decrowding and decompensating skeletal class III in both upper and lower arches.

2. Surgical phase: It involved Le Fort I osteotomy for forward positioning of maxilla by $5 \mathrm{~mm}$, bilateral sagittal split osteotomy (BSSO) for mandibular setback by $5 \mathrm{~mm}$ and a vertical reduction augmentation genioplasty.

3. Post-surgical orthodontics phase: Final settling of occlusion and retention plan.

\section{Treatment}

The essential steps in pre-surgical orthodontics are to align the arches or arch segments and make them 
compatible, and establish the anteroposterior and vertical position of the incisors. Pre-surgical orthodontics was begun with bonding of 0.022 "X0.028" MBT pre-adjusted edgewise prescription appliance. To achieve sufficient decompensation and ideal maxillary and mandibular incisor inclination, therapeutic extractions of upper and lower first premolars were done which was followed by controlled retraction of maxillary and mandidular anterior segment. Lower third molars were also extracted to facilitate the orthognathic surgery later on. Initial leveling and alignment was carried out using 0.016" NiTi, 0.016" X $0.022 " \mathrm{NiTi}$ and $0.019 " \mathrm{X} 0.025 " \mathrm{NiTi}$ followed by 0.019 "X 0.025 " SS for retraction of U/L anteriors and $0.021 " \mathrm{X} 0.025 " \mathrm{SS}$ as final stabilizing wires (Fig. 3). Pre-surgical phase radiographic records were repeated and compared (Fig. 3). Impressions were also taken and models were hand articulated for examining occlusal compatibility. The final stabilizing wires were placed four weeks prior so that they are passive when the impressions are taken for fabrication of surgical splints. This ensures that there will be no tooth movement that could result in poorly fitting splints. Following this, Cephalometric prediction tracing was done.

The maxillary and mandibular dental casts were mounted on a semi adjustable articulator with the help of a face bow transfer (Fig. 4). The individual dental casts were repositioned, simulating the movements of the jaws. An intermediate acrylic occlusal splint was fabricated after the maxillary cast was advanced on the articulator. The mandibular cast was then repositioned to oppose the maxillary cast, simulating the final position of the jaws at surgery. Based on this position the final occlusal splint was then fabricated (Fig. 4), which was reviewed by both Orthodontist and the Surgeon together.

Le- Forte I surgical procedure was carried out as decided and the maxilla was repositioned $5 \mathrm{~mm}$ anteriorly. Bilateral sagittal split osteotomy was performed and the mandible was set back by $5 \mathrm{~mm}$ along with vertical reduction augmentation genioplasty. Rigid type fixations were used in both jaws using mini plates and screw on both sides (Fig. 5). The patient was hospitalized for 2 days and was advised to maintain a soft diet for the first 2 weeks after surgery. By 8 weeks, patient was back on a normal diet.

Once initial healing was satisfactory and patient had attained an average range of motion, active orthodontic treatment was resumed. Post-surgical intra oral photographs (Fig. 6) and radiographs (Fig. 7) were taken. The occlusal splint was removed and the heavier 0.021 " X 0.025 " SS wires were replaced with smaller dimension 0.017 " X 0.025" TMA in the upper arch and 0.016" SS round wire in the lower arch. Bilateral posterior box elastics with anterior vertical elastics were given. The patient was instructed to wear elastics full time for the next few weeks which were weaned off as further detailing of occlusion was taking place. After five months of active post-surgical orthodontic treatment, fixed appliances were debonded (Fig. 8) and post treatment retention phase was initiated with fixed lingual retainers in both $\mathrm{U} / \mathrm{L}$ arches. Post treatment lateral cephalogram was evaluated for treatment changes by superimposition (Fig. 9).
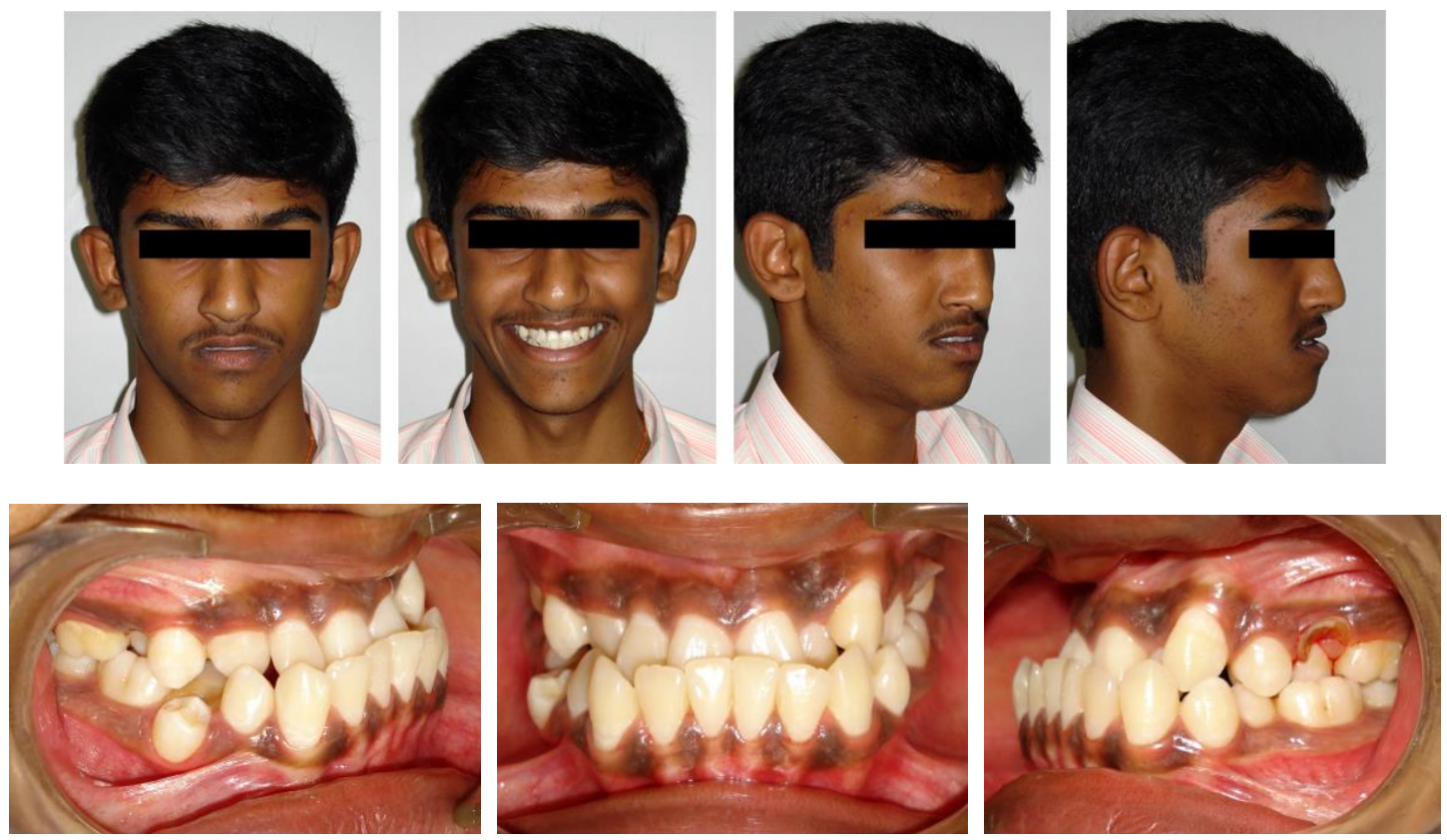

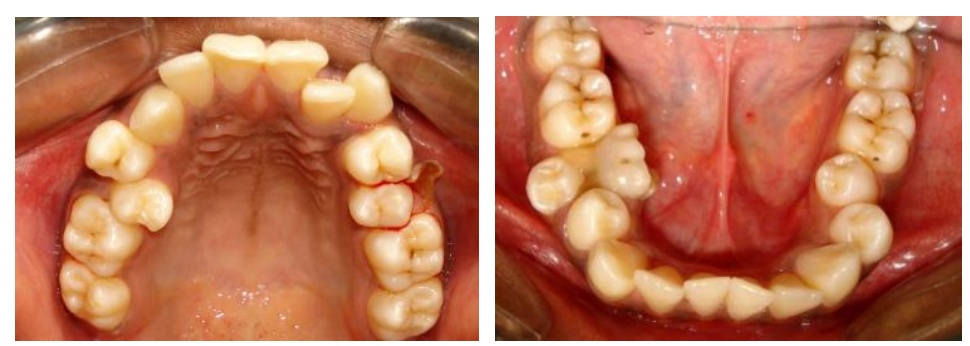

Fig. 1: Pre-treatment photographs

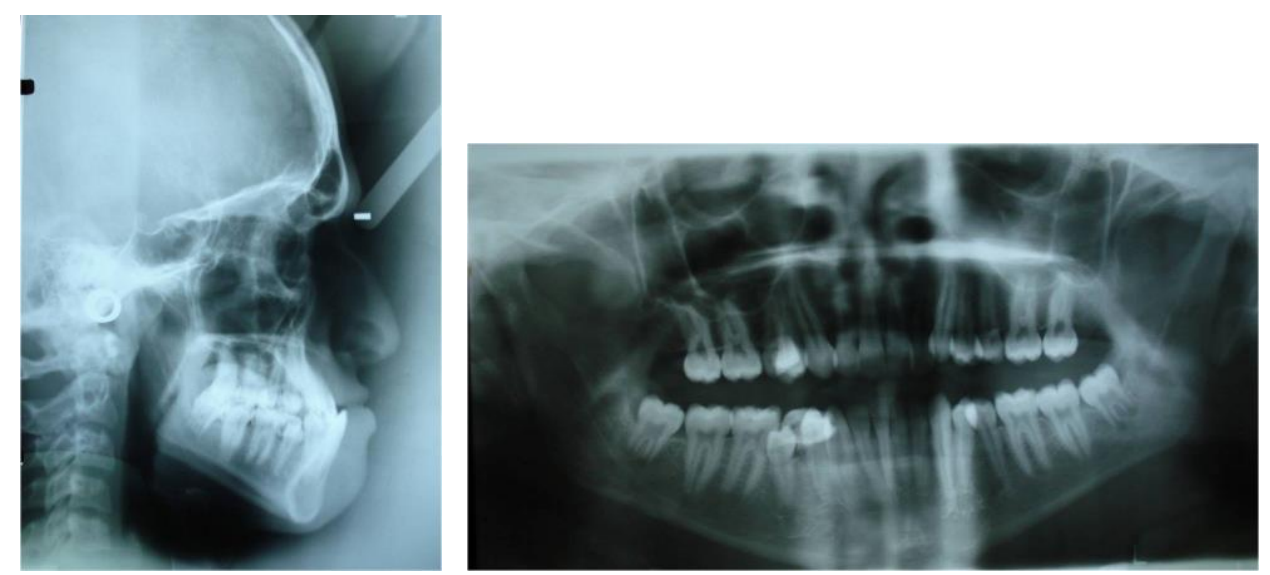

Fig. 2: Pre-treatment OPG and lateral cephalogram
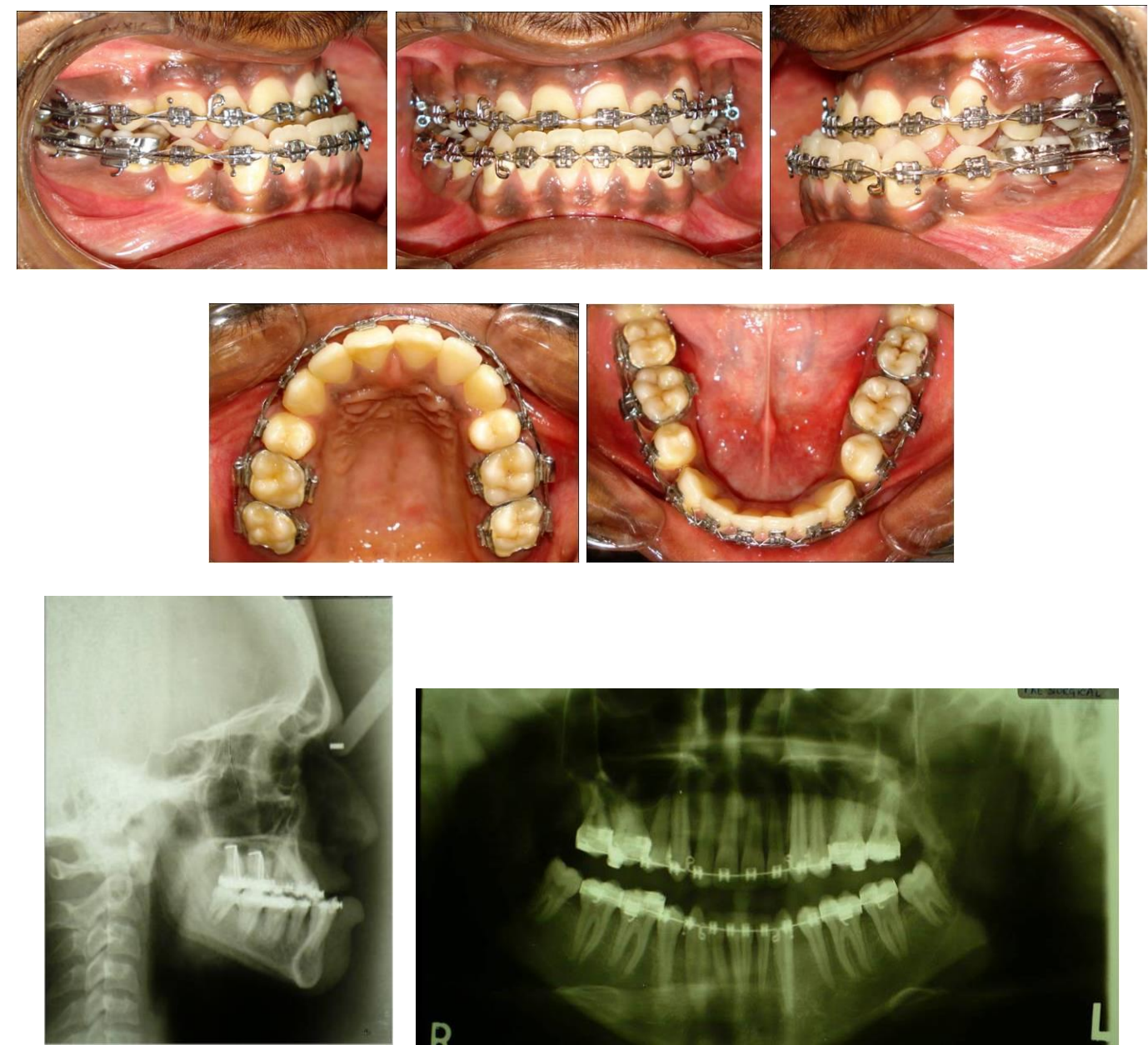

Fig. 3: Pre-Surgical orthodontic phase - decompensation 

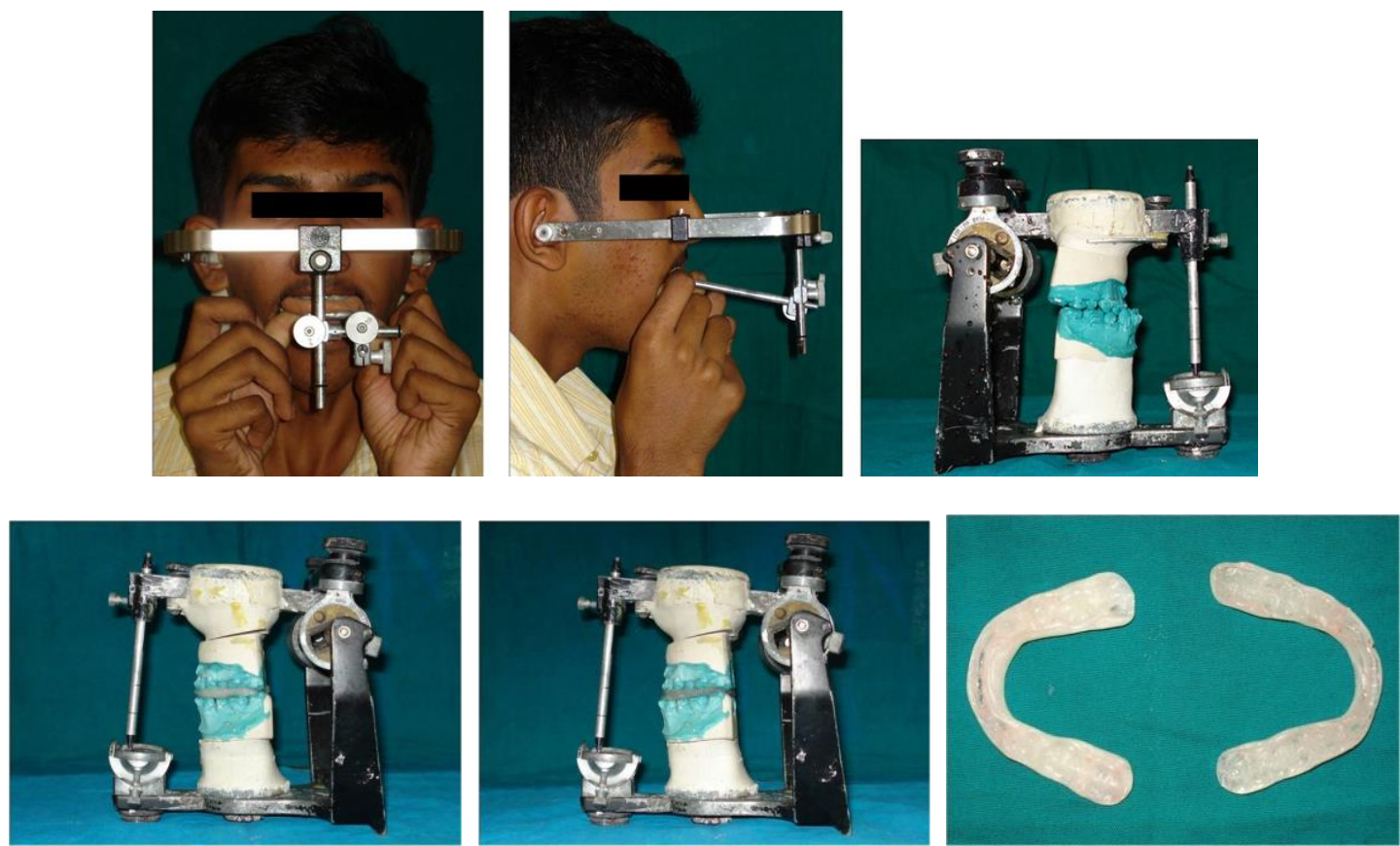

Fig. 4: Face bow transfer to Semi-adjustable articulator $\&$ splint fabrication
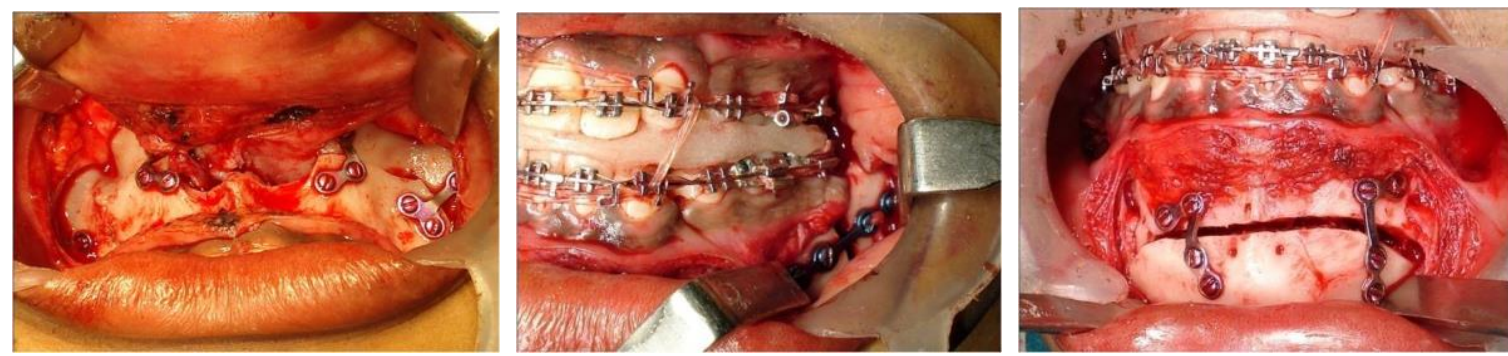

Fig. 5: Maxillary advancement, Mandibular setback and Vertical reduction augmentation genioplasty
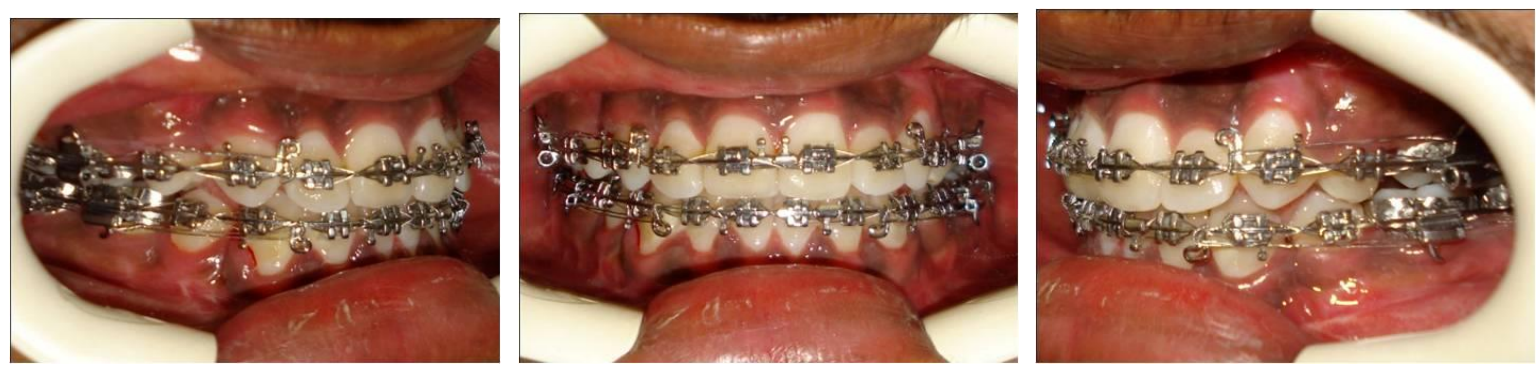

Fig. 6: Post-surgical Intraoral photographs
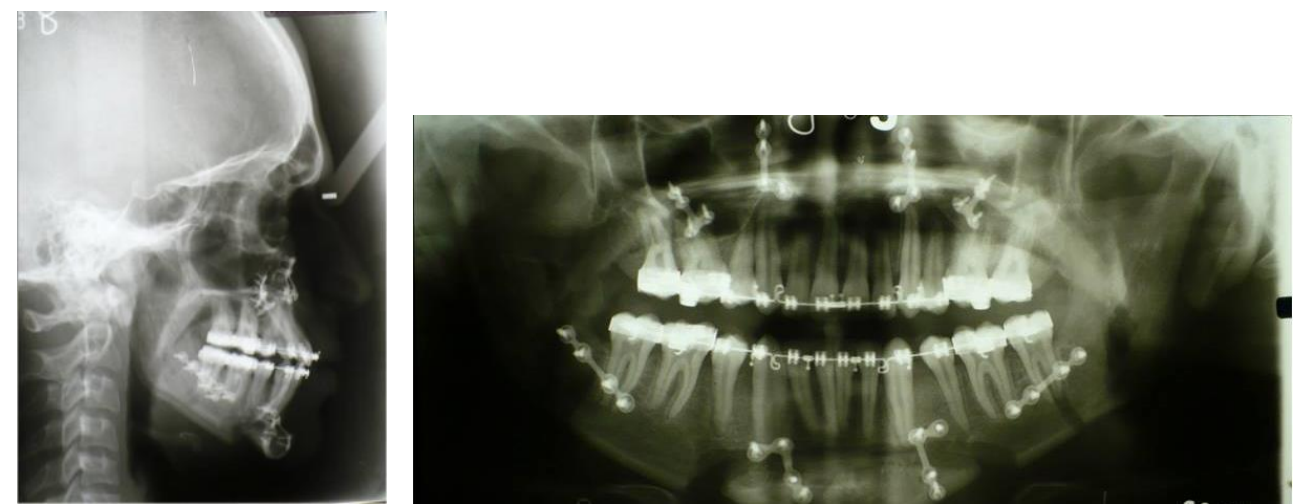

Fig. 7: Post-surgical radiographs 

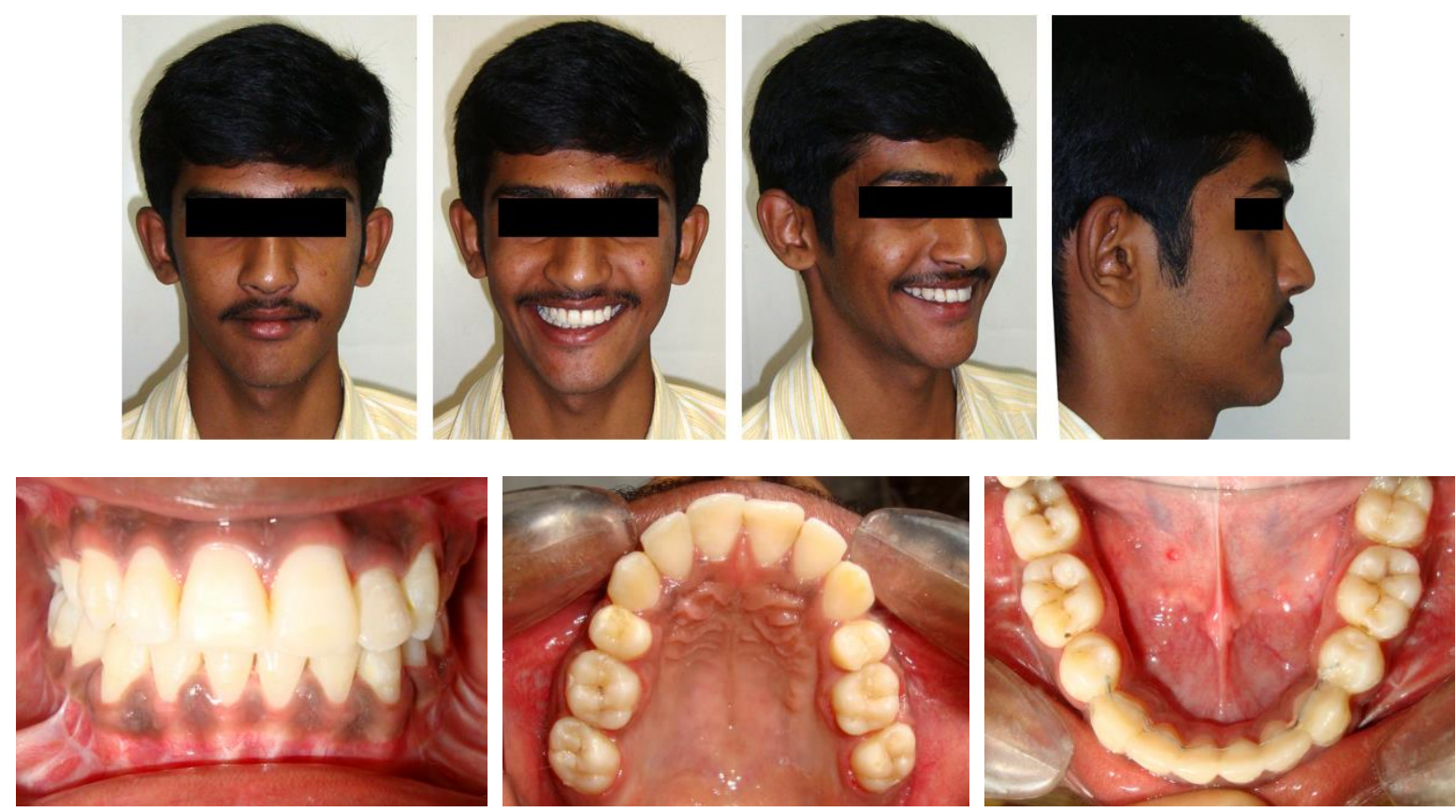

Fig. 8: Post-treatment photographs

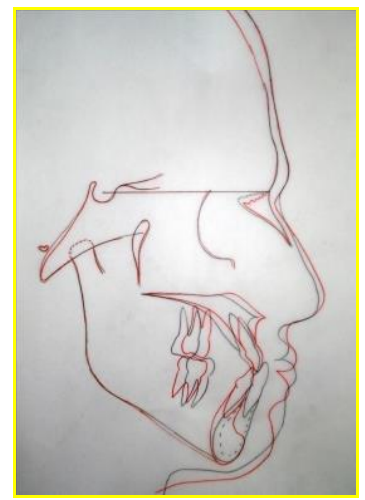

Fig. 9: Pre and post-treatment superimposition

Table 1: Pre and post treatment cephalometric appraisal

\begin{tabular}{|l|c|c|c|c|}
\hline & Norms & Pre-treatment & Pre-Surgical & Post-Treatment \\
\hline SNA & $82^{0}$ & $78^{0}$ & $78^{0}$ & $81.5^{0}$ \\
\hline SNB & $80^{0}$ & $85^{0}$ & $85^{0}$ & $79^{0}$ \\
\hline ANB & $2^{0}$ & $-7^{0}$ & $-7^{0}$ & $2.5^{0}$ \\
\hline U1-NA & $22^{0} / 4 \mathrm{~mm}$ & $31^{0} / 7 \mathrm{~mm}$ & $23^{0} / 3 \mathrm{~mm}$ & $23^{0} 3 \mathrm{~mm}$ \\
\hline L1-NB & $25^{0} / 4 \mathrm{~mm}$ & $16^{0} / 5 \mathrm{~mm} \mathrm{~mm}$ & $21^{0} / 4 \mathrm{~mm}$ & $21^{0} / 4 \mathrm{~mm}$ \\
\hline GoGn-SN & $32^{0}$ & $35^{0}$ & $36^{0}$ & $33^{0}$ \\
\hline IMPA & $90^{0}$ & $81^{0}$ & $89^{0}$ & $89^{0}$ \\
\hline Co-Gn & $126+/-4 \mathrm{~mm}$ & $130 \mathrm{~mm}$ & $130 \mathrm{~mm}$ & $126 \mathrm{~mm}$ \\
\hline Co-Pt A & $98+/-4 \mathrm{~mm}$ & $90 \mathrm{~mm}$ & $90 \mathrm{~mm}$ & $95 \mathrm{~mm}$ \\
\hline A-N Perp & $0 \mathrm{~mm}$ & $-5.5 \mathrm{~mm}$ & $-5.5 \mathrm{~mm}$ & $-1 \mathrm{~mm}$ \\
\hline Pog-N Perp & 0 to $-4 \mathrm{~mm}$ & $2 \mathrm{~mm}$ & $2 \mathrm{~mm}$ & $-1.5 \mathrm{~mm}$ \\
\hline
\end{tabular}

\section{Results}

Treatment objectives of attaining class I molar and canine relationship bilaterally with normal overjet and overbite, improved patient profile and smile esthetics along with balanced facial soft tissues were achieved.
Pre and post cephalometric values have been compared in Table 1.

\section{Discussion}

This case report describes the management of an adult male patient with severe skeletal and dental class 
III malocclusion. Angle's class III skeletodental relationship is one of the most complicated problems in both the childhood and adulthood of all the dentofacial abnormalities. ${ }^{7}$

Though borderline cases can often be treated with orthodontics alone, patients with significant skeletal class III discrepancies are often treated with unijaw or bijaw orthognathic surgery along with orthodontic appliance treatment. ${ }^{8}$ It is generally accepted that the prime benefits of orthognathic treatment are likely to be psychosocial in nature and that majority of the patients seek treatment because of concerns about their dentofacial esthetics. ${ }^{9}$

Post-surgical orthodontics was done in this case for five months, and it primarily involved detailing of the occlusion and permanent retention. The duration of the post-surgical orthodontic phase depends on the degree of preparation achieved during pre-surgical orthodontic phase $^{10}$. Long term stability of achieved results is one of the major goals of orthodontic treatment. Permanent retention is increasingly being recommended as the only way to ensure long-term stability of an orthodontic treatment result. Proper goals of treatment, careful mechanotherapy, precise occlusal equilibration, and well-chosen retention procedures play a role in achieving occlusal homeostasis.

\section{Conclusion}

The combination of surgery and orthodontic treatment makes it possible to treat dentofacial deformities that previously could not have been corrected orthodontically alone. The analysis and management of facial esthetics are extremely complex issues. Newer surgical techniques have broadened the scope of Orthodontist's treatment capability. The case presented is a classic example of this situation, wherein, orthodontic possibilities are limited and requires a team work with an oral surgeon. Technological advances in the field of diagnosis and treatment modalities have helped the orthodontic-surgical team to achieve more difficult treatment goals to overall enhance the quality of life of patients with such facial skeletal disharmonies.

\section{References}

1. Reyneke JP. Essentials of Orthognathic Surgery, Quintessence, Carol Stream, Ill, USA, 2003.

2. H.L. Legan. Orthodontic considerations of orthognathic surgery, in Principles of Oral and Maxillofacial Surgery, L. J.Peterson, Ed.,vol.3,pp.1237-1278. Lippincott, Philadelphia, Pa, USA, 1992.

3. Lew KK, Foong WC. Horizontal skeletal typing in an ethnic Chinese population with true class III malocclusion. Br J Orthod. 1993;20:19-23.

4. Kharbanda OP, Sidhu SS, Sundaram KR, Shukla DK. Prevelance of malocclusion and its traits in Delhi children. J Indian Orthod Soc. 1995;26:98-103.

5. Ishii N, Deguchi T, Hunt N. Craniofacial difference between Japanese and British Caucasian females with a skeletal class III malocclusion. Eur J Orthod. 2002;24:493-9.

6. Hunt ND, Rudge SJ. Facial profile and orthognathic surgery. Br J Orthod. 1984;11:126-36.

7. Wilmot DR. Soft tissue profile changes following correction of class III malocclusion by mandibular surgery. Br J Orthod. 1981;8(7):175-181.

8. Tompach PC, Wheeler JJ, Fridrich KL. Orthodontic considerations in orthognathic surgey. Int J Adult Orthodontics and Orthognathic Surgery. 1995;10:97-107.

9. Stirling J, Latchford G, Morris DO. Elective orthognathic treatment decision-making: a survey of patient reasons and experiences. J Orthod. 2007;34:113-127.

10. Luther F, Morris DO, Karnezi K. Orthodontic treatment following orthognathic surgery: how long does it take and why? A retrospective study. Journal of Oral and Maxillofacial Surgery. 2007;65:10,1969-1976. 